\title{
INFLUENCE OF SAMPLE ROTATION ON THE SHAPE OF THE FREE INDUCTION DECAY*
}

\author{
B. Blicharska and D. KRUK \\ Instilute of Physics, Jagiellonian University, Reymonta 4, 30-059 Kraków, Poland \\ (Received February 8, 1993; in final form June 22, 1993)
}

Free induction decay is calculated at the presence of rotation of a liquid sample in a static magnetic field with a constant gradient.

PACS numbers: $76.60 .-\mathrm{k}$

Sample rotation is commonly used in NMR as one of the methods which improve the effective homogeneity of magnetic fields. The magic angle spinning (MAS), the double rotation (DOR) and the dynamic angle spinning (DAS) can remove line broadening of an NMR spectrum in solids [1,2].

It is generally assumed that the NMR spectrum of a rotating sample (in the case of isotropic liquids) is simply the orientationally a veraged spectrum [3]. Our calculations, which are presented below, show that the sample rotation can change the shape of free induction decay (FID) (and consequently NMR line). We discuss this fact because, as we shall show later, it can disturb the absolute evaluation of the measured relaxation time.

The shape of the FID can be calculated from the well-known Bloch equation for the transverse component of the magnetization $M$ (without diffusion) under following assumptions [4]:

$$
\boldsymbol{B}=\left(B_{x}, B_{y}, B_{z}\right), \quad B_{x}=B_{y}=0, \quad B_{z}=B_{o}+G * r, \quad G(t)=\text { const }
$$

where $B_{0}$ is a static magnetic field and $G$ is the constant gradient of the magnetic field $\boldsymbol{B}$ (natural magnetic field inhomogeneity or artificially produced gradient)

$$
\frac{\mathrm{d} m_{p}}{\mathrm{~d} t}=\gamma\left(m \times B_{\mathrm{ef}}\right)_{p}-\frac{m_{p}}{T_{2}} \quad(p=x, y),
$$

where

$$
M_{p}=\int_{V} m_{p} \mathrm{~d} V
$$

${ }^{*}$ This work was supported by the research grant of the Committee for Scientific Research 224119102. 
After transformation of the above Eq. (1) into rotating frame $x^{\prime} y^{\prime} z^{\prime}$ which rotates with resonant frequency $\omega_{0}=\gamma B_{0}$ around $z$ axis we obtain

$$
M_{\perp}^{\prime}=m_{0} \mathrm{e}^{t / T_{2}} \int_{V} \mathrm{e}^{-\mathrm{i} \gamma \int_{0}^{1}(G * r) \mathrm{d} t} \mathrm{~d} V
$$

where $M_{\perp}^{\prime}=M_{x}^{\prime}+\mathrm{i} M_{y}^{\prime}$ in the rotating frame and $m_{0}=M_{0} / V$ is the density of magnetization of the sample in the thermodynamic equilibrium.

For a cylindrical sample, which is commonly used in such experiments, it is convenient to integrate in the cylindrical frame $(\rho, \phi, z)$ :

$$
M_{\perp}^{\prime}(t)=m_{0} \mathrm{e}^{-t / T_{2}} \int_{0}^{R} \int_{-l / 2}^{l / 2} \int_{0}^{\pi / 2} \mathrm{e}^{-\mathrm{i} \gamma \int_{0}^{t}\left(G_{*} r\right) \mathrm{d} t} \rho \mathrm{d} \rho \mathrm{d} \phi \mathrm{d} z .
$$

Let the sample rotate around the axis perpendicular to $B_{0}$ with frequency $\omega_{R}$.

In consequence, the value of $G * r$ becomes dependent on time

$$
\boldsymbol{G} * \boldsymbol{r}=G_{\|} z+G_{\perp} \rho \cos \left(\omega_{R} t+\phi\right)
$$

where $G_{\|}$and $G_{\perp}$ are parallel and transverse components of the gradient, respectively.

After integration over time we obtain

$$
G_{\perp} \rho(t)=\int_{0}^{t} G_{\perp} \rho \cos \left(\omega_{R} t^{\prime}+\phi\right) \mathrm{d} t^{\prime}=-\frac{G_{\perp} \rho}{\omega_{R}}\left[\sin \left(\omega_{R} t+\phi\right)-\sin \phi\right]
$$

and the transverse magnetization is equal to

$$
\begin{aligned}
M_{\perp}(t)= & m_{0} \mathrm{e}^{-t / T_{2}} \int_{0}^{R} \int_{-l / 2}^{l / 2} \int_{0}^{2 \pi} \exp \left[\left(\frac{-\mathrm{i} \gamma G_{\perp} \rho}{\omega_{R}}\right) 2 \cos \left(\omega_{R} t / 2+\phi\right)\right. \\
& \left.\times \sin \left(\omega_{R} t / 2\right)\right] \exp \left(-\mathrm{i} \gamma G_{\|} t z\right) \rho \mathrm{d} \rho \mathrm{d} \phi \mathrm{d} z .
\end{aligned}
$$

After expansion in power series form we obtain

$$
M_{\perp}(t)=M_{0} \mathrm{e}^{-t / T_{2}} \sin c\left(\gamma G_{\|} l l / 2\right) \sum_{k=0}^{\infty} \frac{(-1)^{k}}{k !(k+1) !}\left(\gamma G_{\perp} R\right)^{2 k} \frac{\sin ^{2 k}\left(\omega_{R} t / 2\right)}{\omega_{R}^{2 k}} .
$$

When $\omega_{R}=0$ (without rotation), Eq. (6) has the form

$$
\begin{aligned}
M_{\perp}(t)= & M_{0} \mathrm{e}^{-t / T_{2}} \frac{2 J_{1}\left(\gamma G_{\perp} R l\right)}{\gamma G_{\perp} R t} \operatorname{sinc}\left(\gamma G_{\|} l l / 2\right) \\
& =M_{0} \mathrm{e}^{-t / T_{2}} \sin c\left(\gamma G_{\|} t l / 2\right) \sum_{k=0}^{\infty} \frac{(-1)^{k}}{k !(k+1) !}\left(\frac{\gamma G_{\perp} R l}{2}\right)^{2 k},
\end{aligned}
$$

where $J_{1}\left(\gamma G_{\perp} R t\right)$ is the Bessel function of first order.

The shape of the FID without the sample rotation calculated from Eq. (7), for two different values of gradient $G_{\perp}$, is presented in Fig. 1. At higher gradient the FID signal becomes narrower ( 2 times when the gradient is increased by factor two) with greater number of oscillations in the "tail" [5].

Figure 2 shows the shape of FID with the sample rotation calculated from Eq. (6). For comparison both FID's with and without rotation in the short time scale are drawn in Fig. 3. 

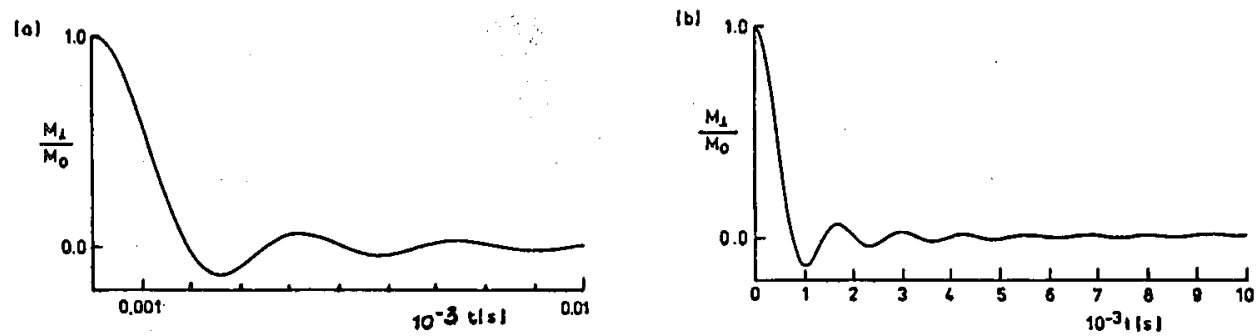

Fig. 1. The shape of FID without rotation calculated for two different gradients $G_{\perp}$ from Eq. (7) for the following parameters: $T_{2}=1 \mathrm{~s}, G_{\|}=0$ and gradient $G_{\perp}$ : (a) $\gamma G_{\perp} R=2 \times 10^{3} \mathrm{rad} / \mathrm{s}$, (b) $\gamma G_{\perp} R=5 \times 10^{3} \mathrm{rad} / \mathrm{s}$.
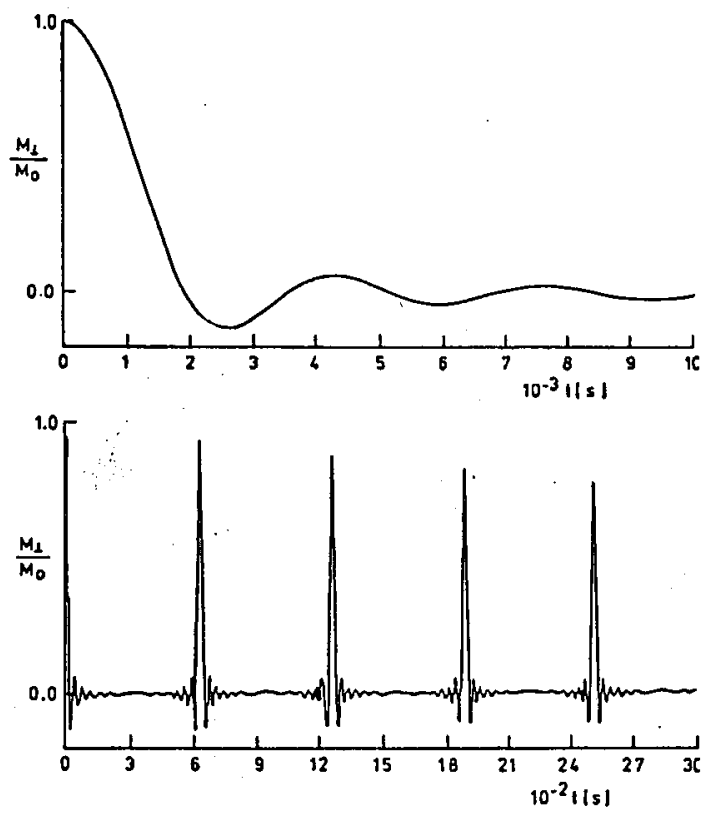

Fig. 2. The shape of FID with sample rotation frequency $\omega_{R}=160 \mathrm{~Hz}$ calculated from Eq. (6) for the following parameters: $T_{2}=1 \mathrm{~s}, \gamma G_{\perp} R=2 \times 10^{3} \mathrm{rad} / \mathrm{s}, G_{\|}=0$.

Let us take a point on the FID, for example, at a very small time $\Delta t=10^{-4} T_{2}$ or $10^{-3} T_{2}$ from the start of FID. When the sample rotation frequency increases, the magnitude of the FID at this increases to achieve a maximum value and then becomes constant (Fig. 4). The value of "critical" frequency (at which the increase in frequency does not change the point position) depends on the value of gradient $G_{\perp}$ and spin-spin relaxation time $T_{2}$ of the sample.

The relaxation measurement is made by recording the decay of magnetization $M$. Since the sample rotation changes the shape of FID (or its height), this can 


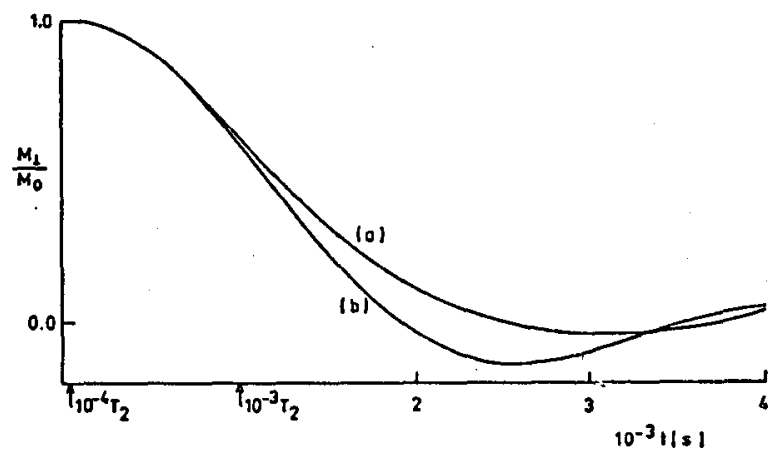

Fig. 3. The comparison of the sliape of FID (a) with and (b) without sample rotation in the short time scale $\left(\omega_{R}=160 \mathrm{Iz}\right.$ and $T_{2}=1 \mathrm{~s}, G_{\|}=0$ and $\left.\gamma G_{\perp} R=2 \times 10^{3} \mathrm{rad} / \mathrm{s}\right)$.
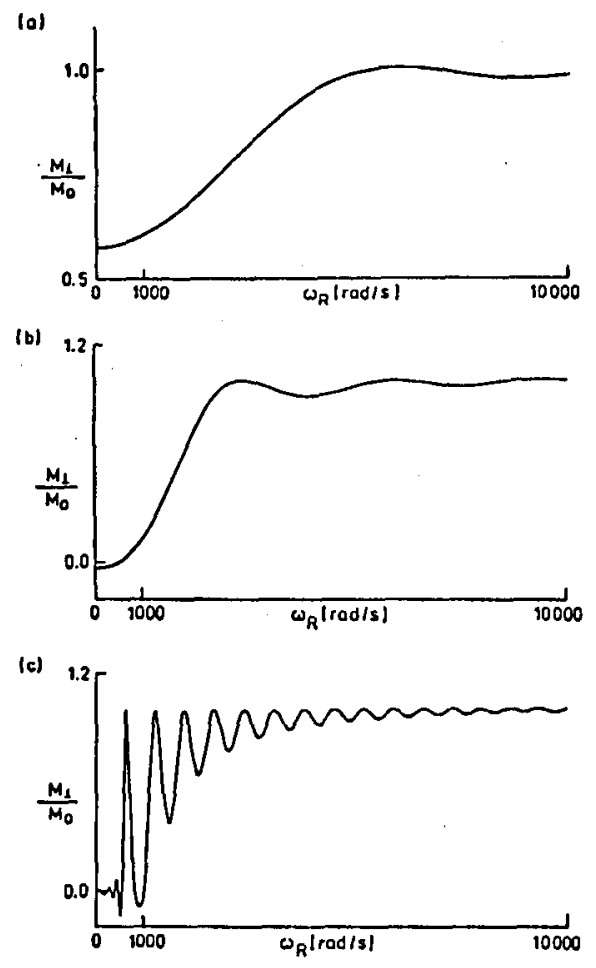

Fig. 4. Dependence of the height of a point on the FID on the sample frequency rotation (a) $\Delta t=10^{-3} T_{2}$, (b) $\Delta t=2 \times 10^{-3} T_{2}$, (c) $\Delta t=10^{-2} T_{2}$.

have an influence on the measured relaxation time. Obviously, the nonstability of sample rotation can be a source of errors.

As mentioned above, the very important factor is the value of spin-spin relaxation time of the sample. For two samples with the same spin-lattice relaxation 
time $T_{1}$ and different spin-spin relaxation time $T_{2}$ the influence of sample rotation can be different (for example for water and methanol with air) [6].

In conclusion, we can say that the sample rotation sometimes changes the shape of the FID. As a consequence, it can affect the measured spin-lattice relaxation time. The observed shortening of $T_{1}$ could therefore be dependent on the gradient of magnetic field and the relaxation time $T_{2}$ of the sample [6].

\section{Reforences}

[1] B.F. Chmelka, A. Pines, Science 246, 71 (1992).

[2] R. Tycko, Phys. Rev. Lett. 58, 2281 (1987).

[3] E.R. Andrew, Prog. Nucl. Magn. Reson. Spectrosc. 8,1 (1971).

[4] A. Abragam, The Principles of Nuclear Magnetism, Clarendon Press, Oxford 1961.

[5] G.A. Williams, H.S. Gutovsky, Phys. Rev. 104, 278 (1956).

[6] B. Blicharska, J. Mol. Liq. 52, 109 (1992). 